\title{
Optimization of Vacuum Pressure Swing Adsorption Processes to Sequester Carbon Dioxide from Coalbed Methane
}

\author{
Daeho Ko*
}

Global Engineering Division of GS Engineering \& Construction, Gran Seoul, 33, Jong-ro, Jongno-gu, Seoul 110-130, Korea

* To whom correspondence should be addressed. Tel: +82 22154 6171, Email: daeho.ko@gsconst.co.kr or daehoko@hotmail.com 


\section{List of Supporting Figure Captions}

Figure S1.1. Operating Step 1 of Four-Bed VPSA

Figure S1.2. Operating Step 2 of Four-Bed VPSA

Figure S1.3. Operating Step 3 of Four-Bed VPSA

Figure S1.4. Operating Step 4 of Four-Bed VPSA

Figure S1.5. Operating Step 5 of Four-Bed VPSA

Figure S1.6. Operating Step 6 of Four-Bed VPSA

Figure S1.7. Operating Step 7 of Four-Bed VPSA

Figure S1.8. Operating Step 8 of Four-Bed VPSA

Figure S1.9. Operating Step 9 of Four-Bed VPSA

Figure S1.10. Operating Step 10 of Four-Bed VPSA

Figure S1.11. Operating Step 11 of Four-Bed VPSA

Figure S1.12. Operating Step 12 of Four-Bed VPSA

Figure S2. Adsorption Isotherms of methane, carbon dioxide, and nitrogen (Ko, 2016)

Figure S3. Pressure Profiles of Pilot Plant Operation Data and Simulation Result

(Operating Steps and Times are shown in Table S2)

Note S1) The adsorption isotherm data of the paper of Ko $(2016)^{5}$ is adopted as shown in Figure S2.

Note S2) The assumption (8) is for the boundary pressure profiles as shown in Figure S3 at the pressure changing step, i.e., steps $4,6,9,10,11$, and 12 as described in the Supporting Information (Table S6.2) of the former paper $(\mathrm{Ko}, 2016)^{5}$.

$$
\begin{aligned}
& P^{\text {step }(K)}{ }_{z=L}=P^{\text {step } K}+\left(P^{\text {step }(K-1)}-P^{\text {step } K}\right) \exp \left(-k^{\text {step } K} t^{\text {step } K}\right) \text { for steps } 4 \text { and } 6 . \\
& P^{\text {step }(K)}{ }_{z=L}=P^{\text {step } K}-\left(P^{\text {step }(K)}-P^{\text {step }(K-1)}\right) \exp \left(-k^{\text {step } K} t^{\text {step } K}\right) \text { for steps } 9,10,11 \text {, and } 12 . \\
& P^{\text {step }(K)}{ }_{z=0}=P^{\text {step } K}+\left(P^{\text {step }(K-1)}-P^{\text {step }(K)}\right) \exp \left(-k^{\text {step } K} t^{\text {ste } K}\right) \text { for steps } 7 \text { and } 8 .
\end{aligned}
$$

The above equations addressed in the assumption (8) are used for the boundary conditions of the time varying pressure profiles.

(5) Ko, D. Development of a Simulation Model for the Vacuum Pressure Swing Adsorption Process To Sequester Carbon Dioxide from Coalbed Methane. Ind. Eng. Chem. Res. 2016, 55 (4), 1013.

Note S3) the unit of purity and recovery in the objective functions of Tables S9 - S10 (equations $37-38$ ) is dimensionless, that is, $0 \leq$ Purity $_{\mathrm{CH} 4} \leq 1$ and $0 \leq$ Recovery $_{\mathrm{CH} 4} \leq 1$. 


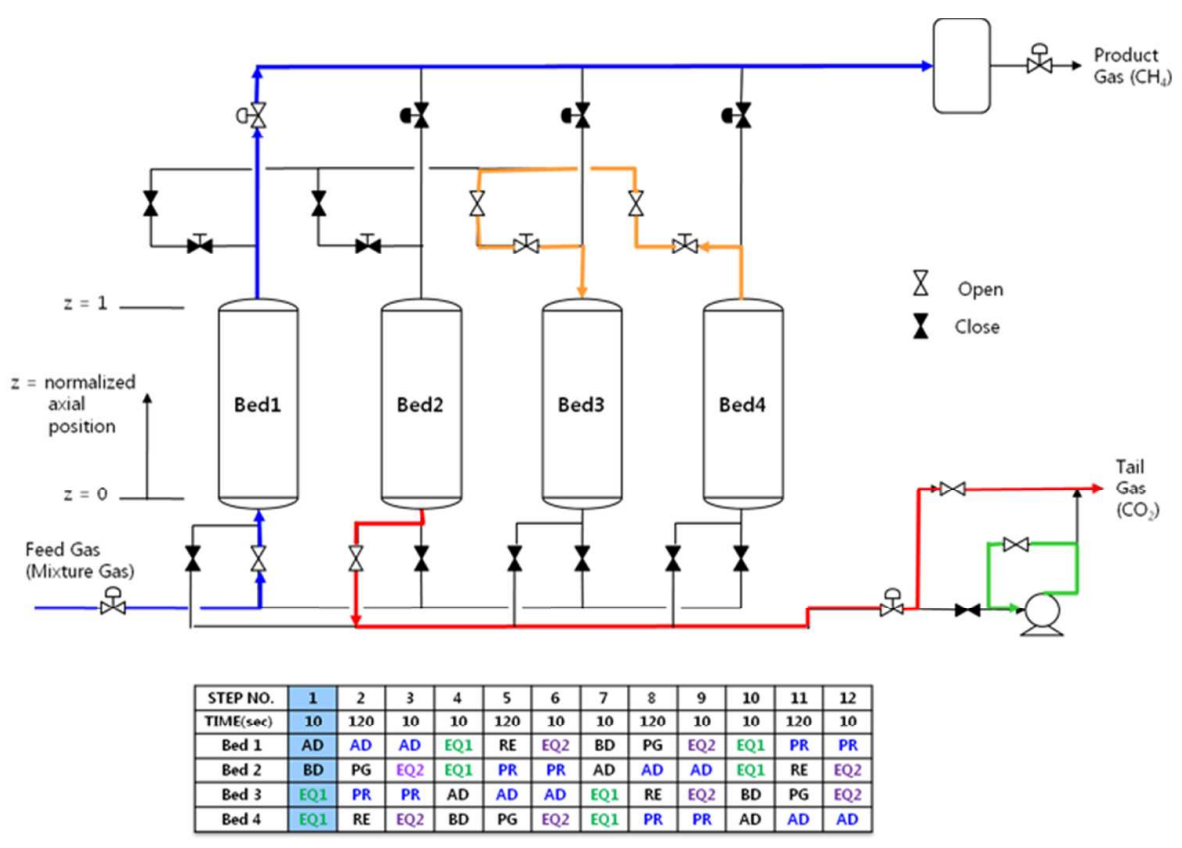

Figure S1.1. Operating Step 1 of Four-Bed VPSA

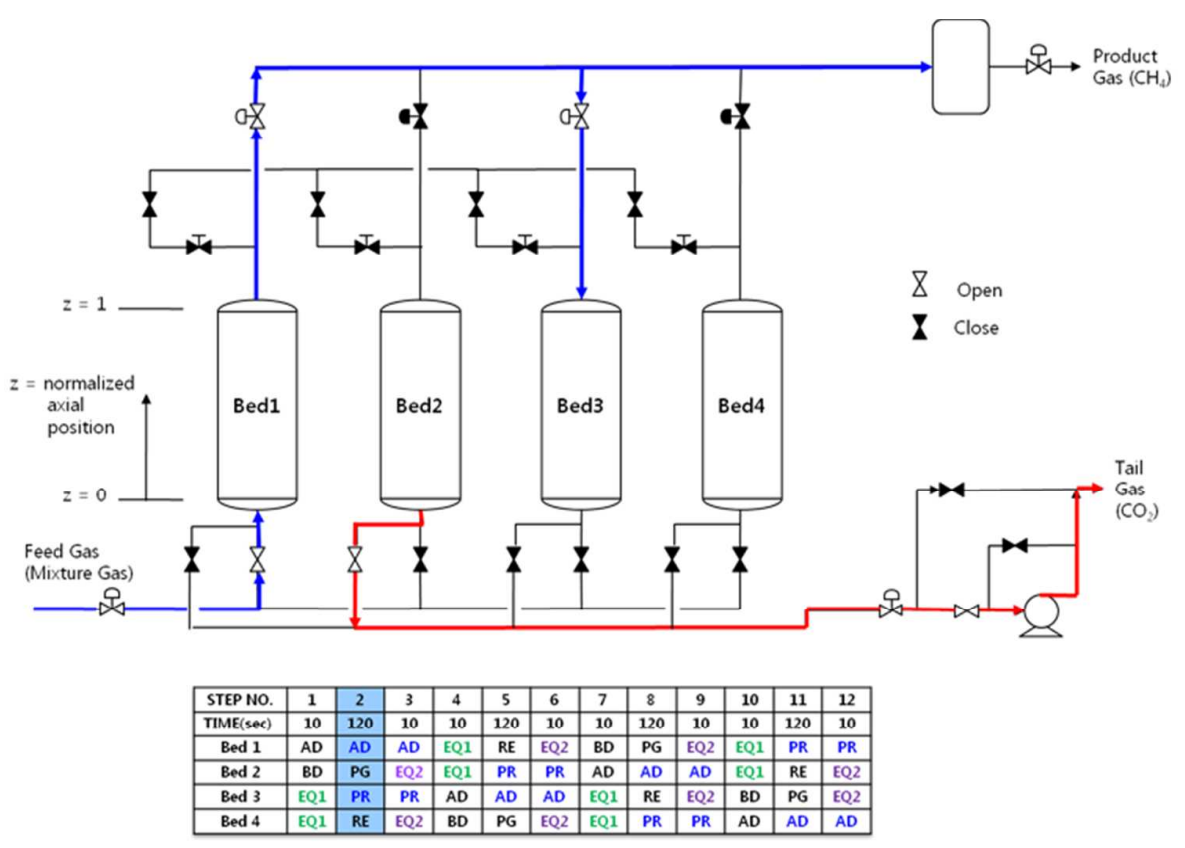

Figure S1.2. Operating Step 2 of Four-Bed VPSA 


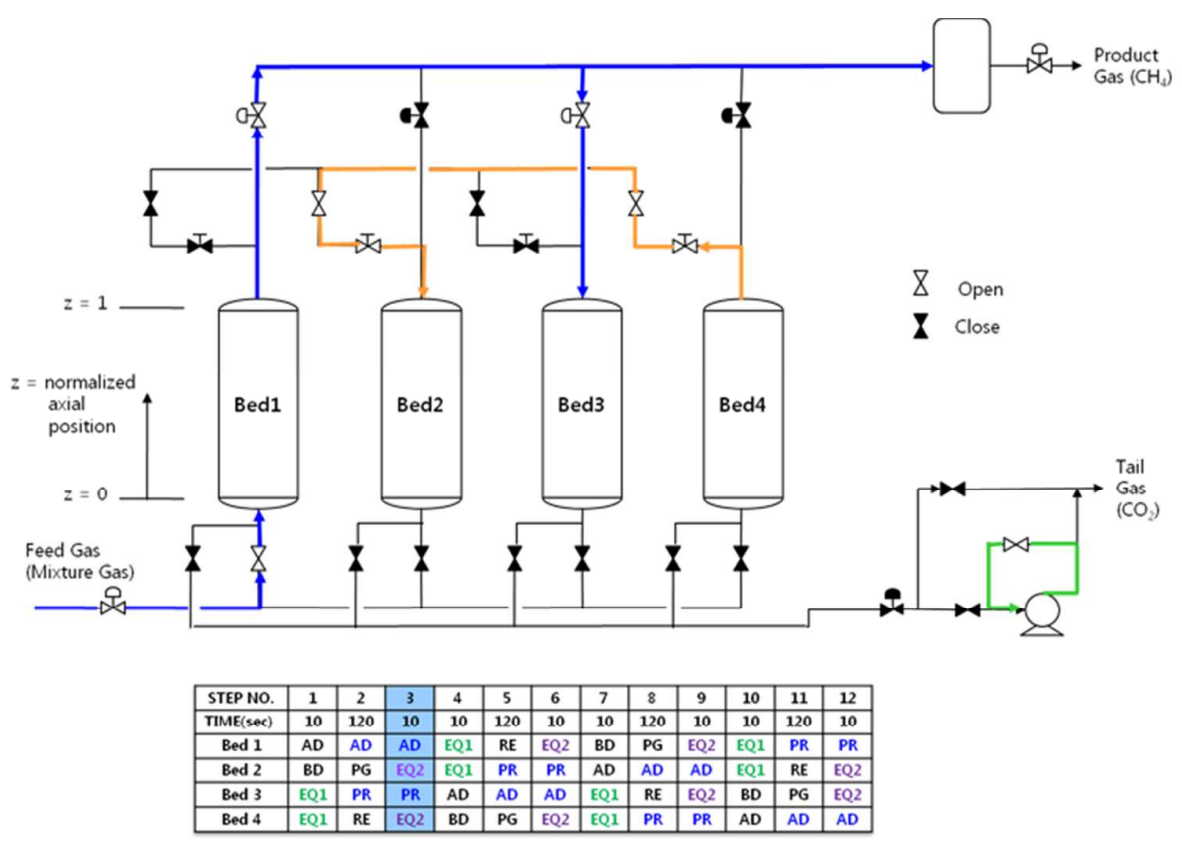

Figure S1.3. Operating Step 3 of Four-Bed VPSA

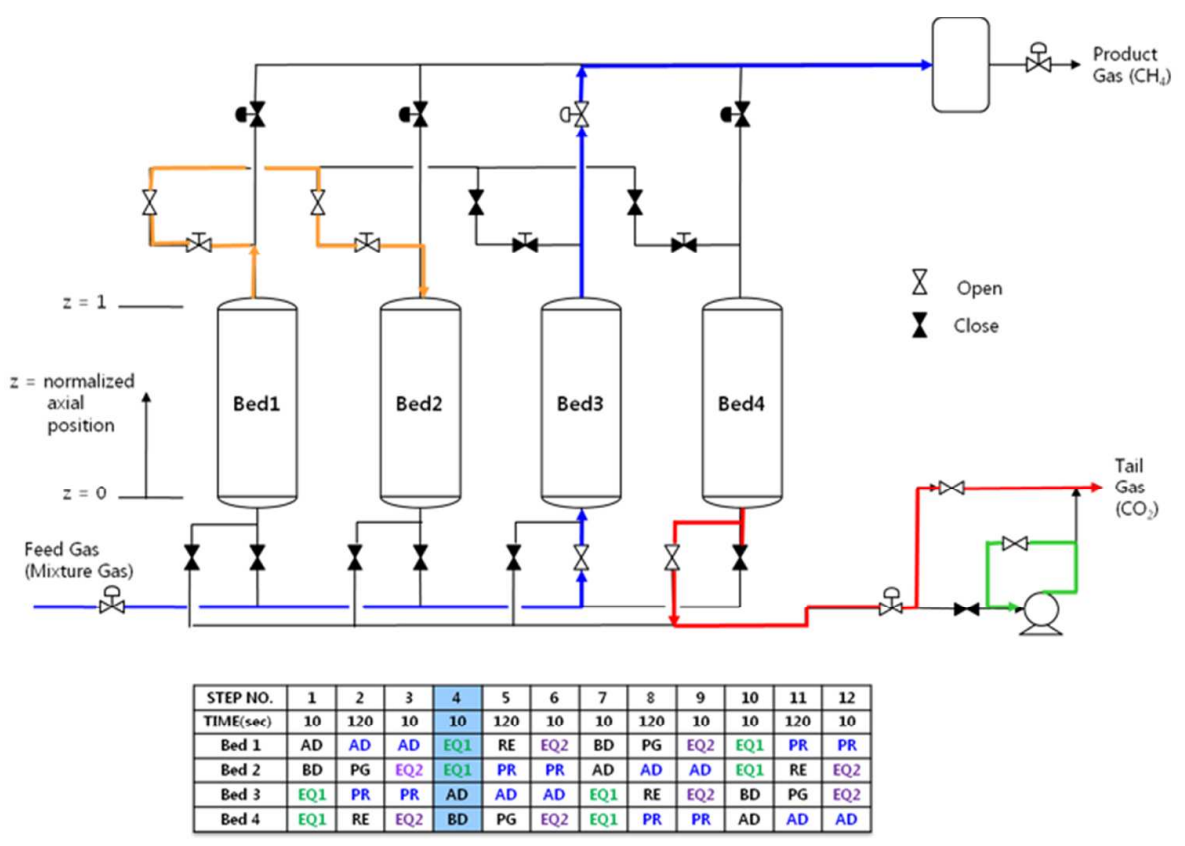

Figure S1.4. Operating Step 4 of Four-Bed VPSA 


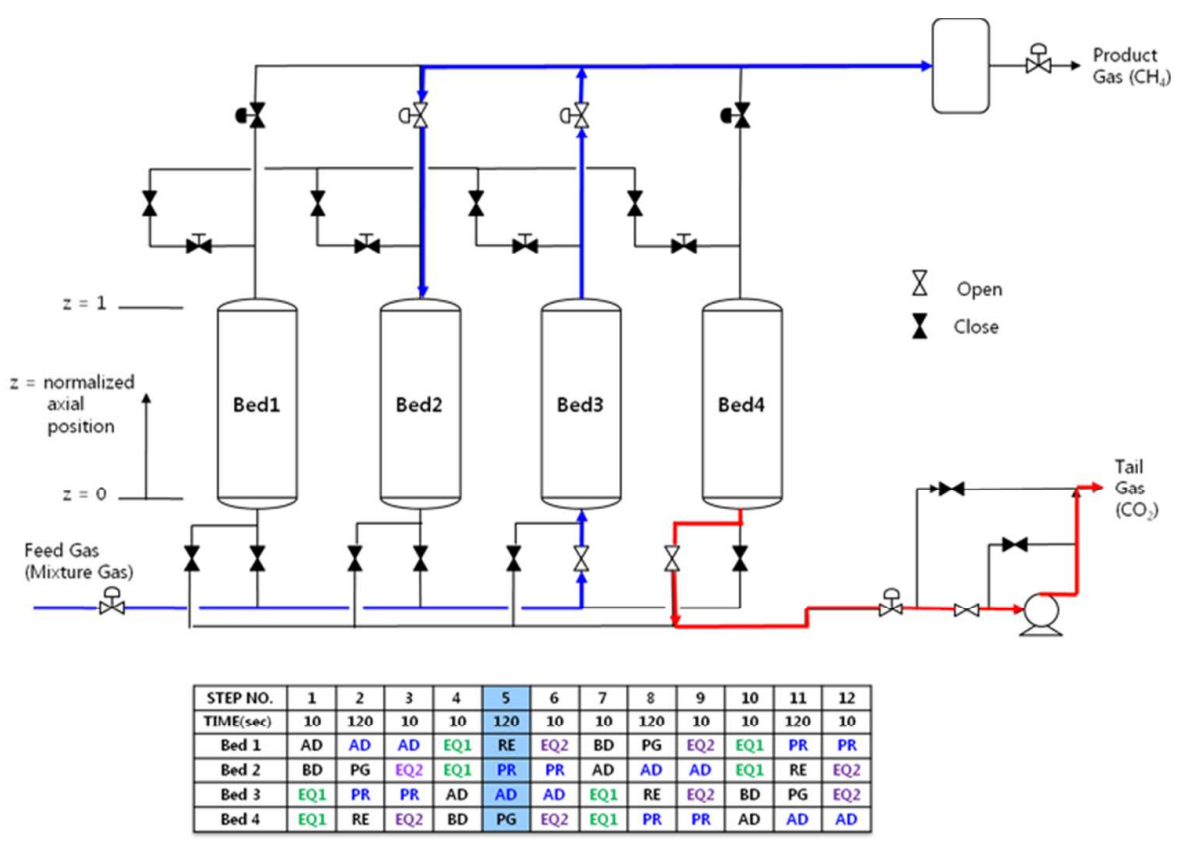

Figure S1.5. Operating Step 5 of Four-Bed VPSA

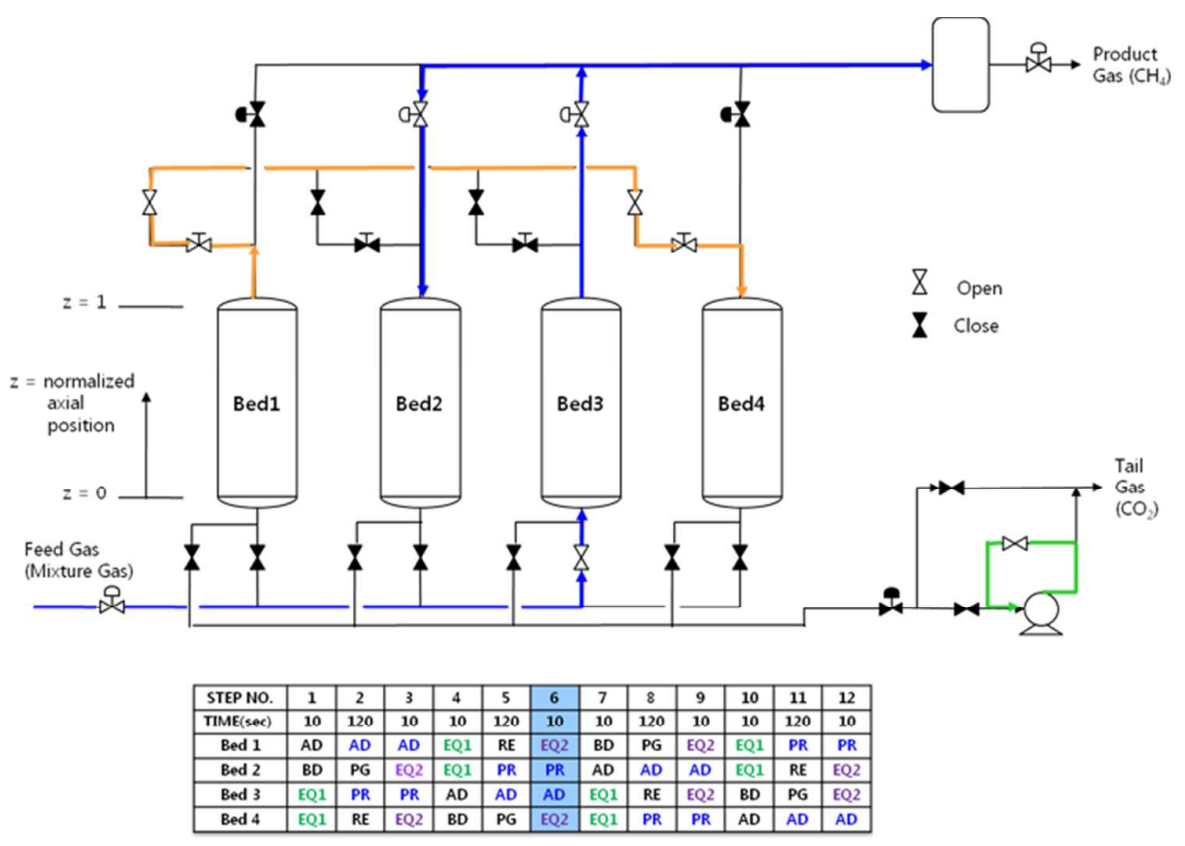

Figure S1.6. Operating Step 6 of Four-Bed VPSA 


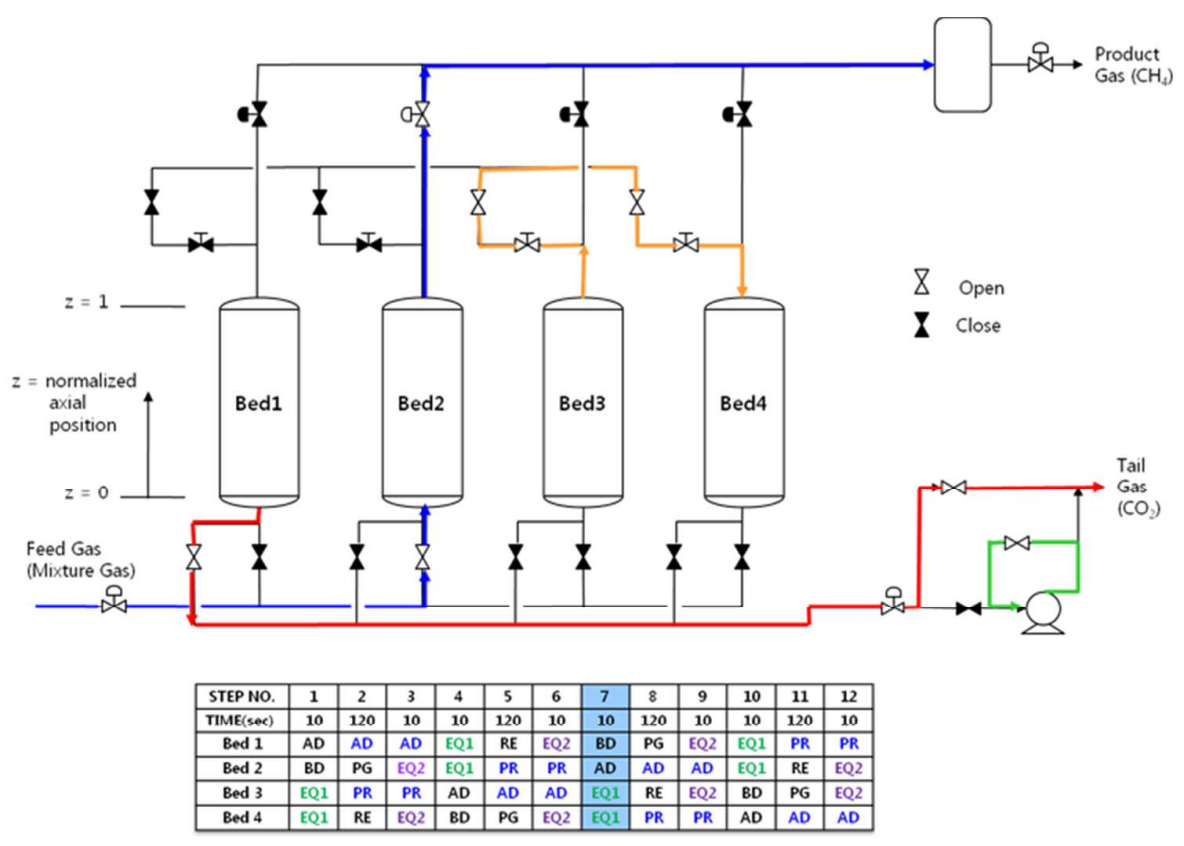

Figure S1.7. Operating Step 7 of Four-Bed VPSA

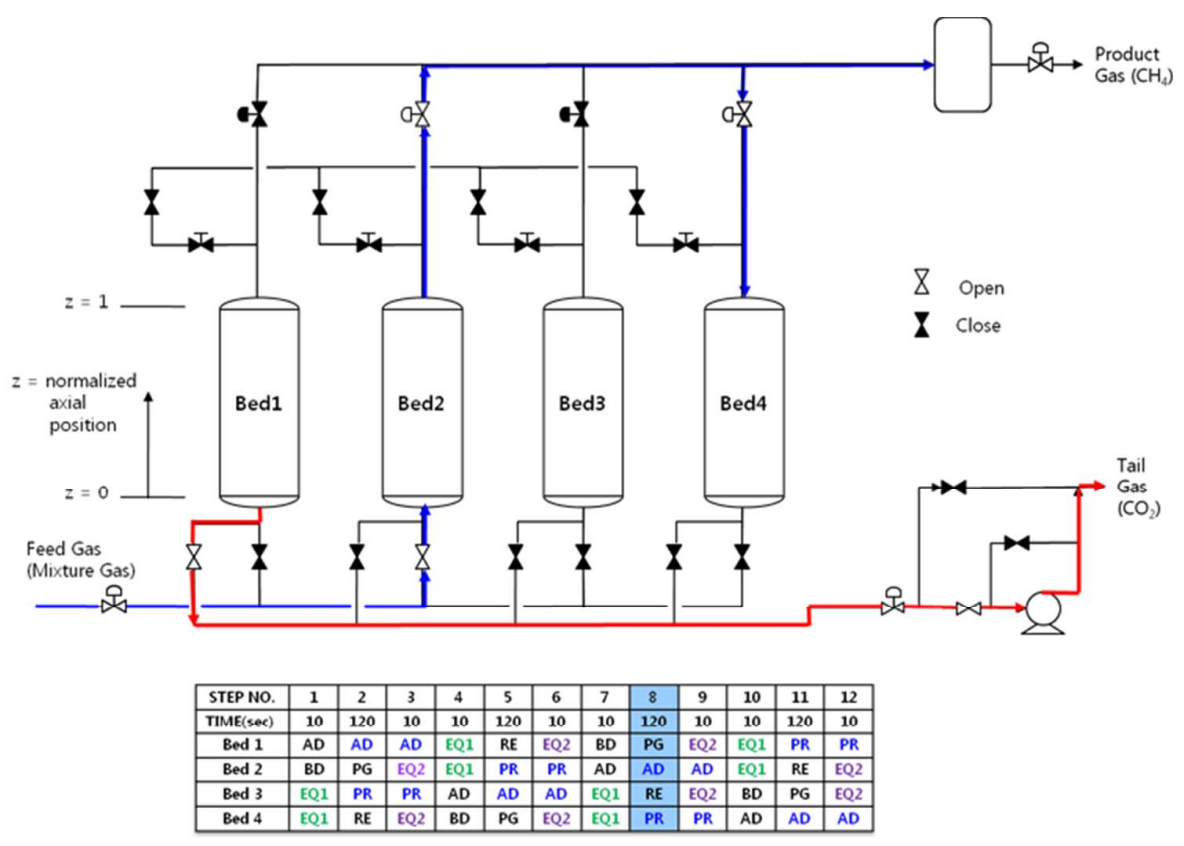

Figure S1.8. Operating Step 8 of Four-Bed VPSA 


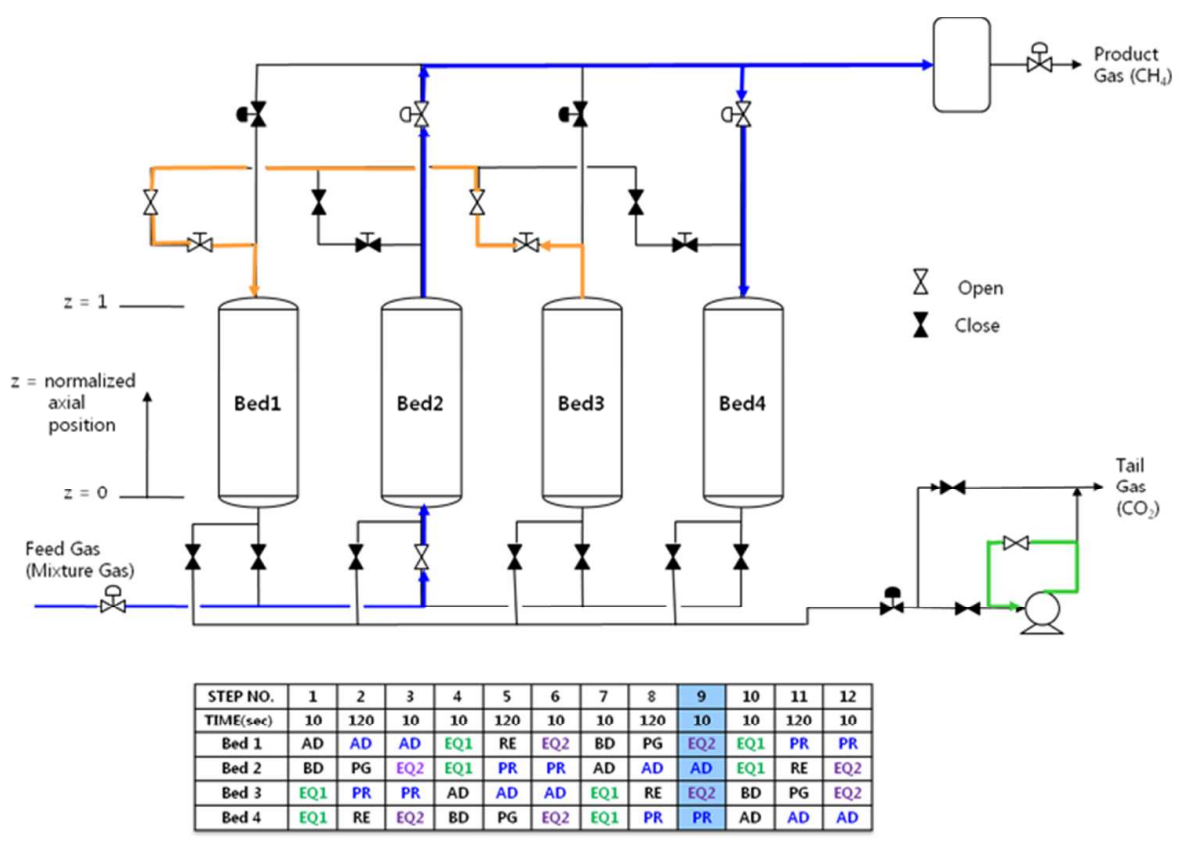

Figure S1.9. Operating Step 9 of Four-Bed VPSA

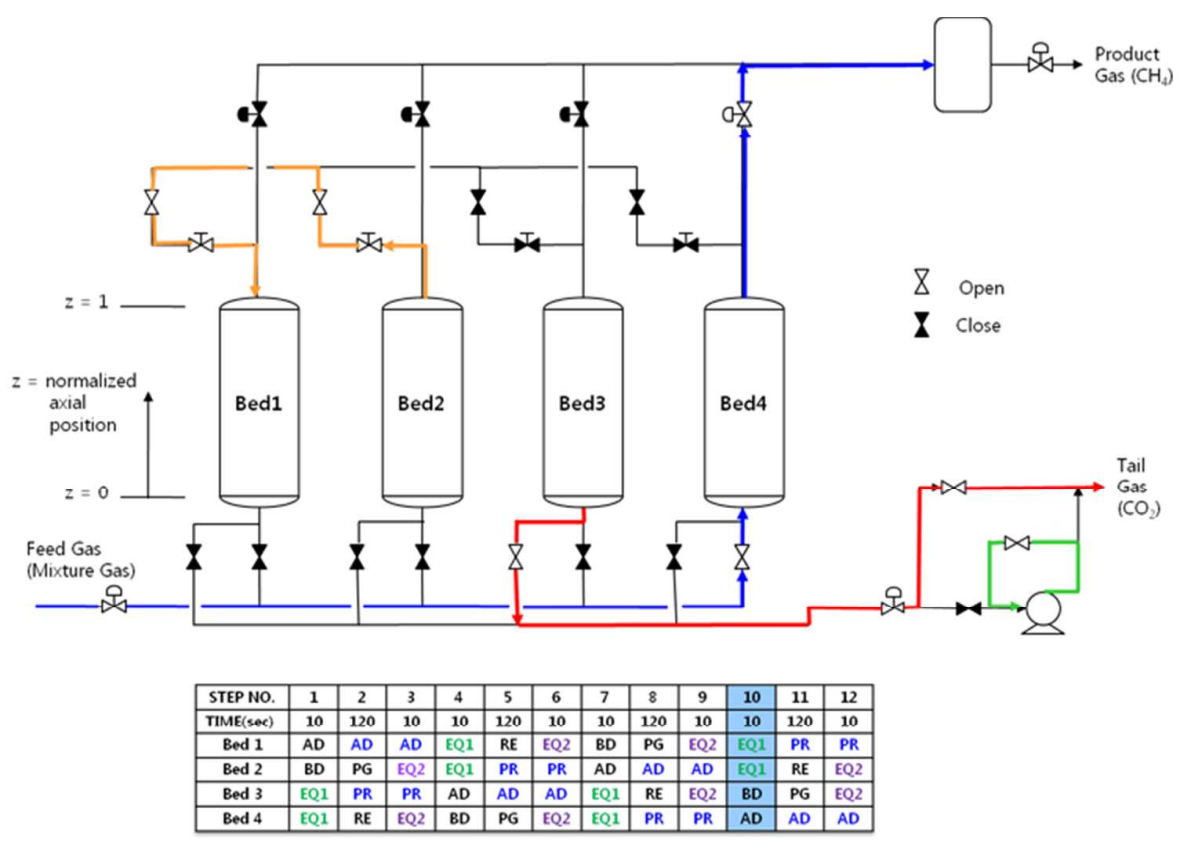

Figure S1.10. Operating Step 10 of Four-Bed VPSA 


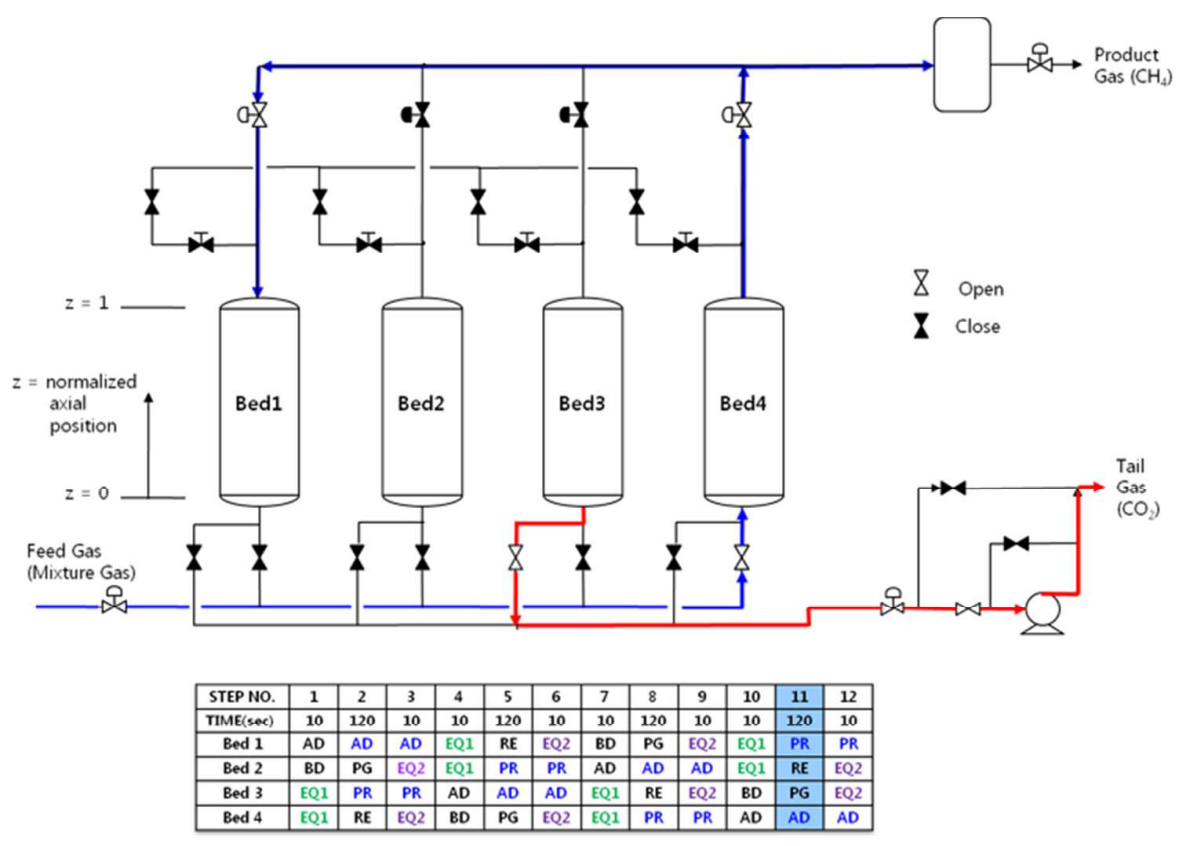

Figure S1.11. Operating Step 11 of Four-Bed VPSA

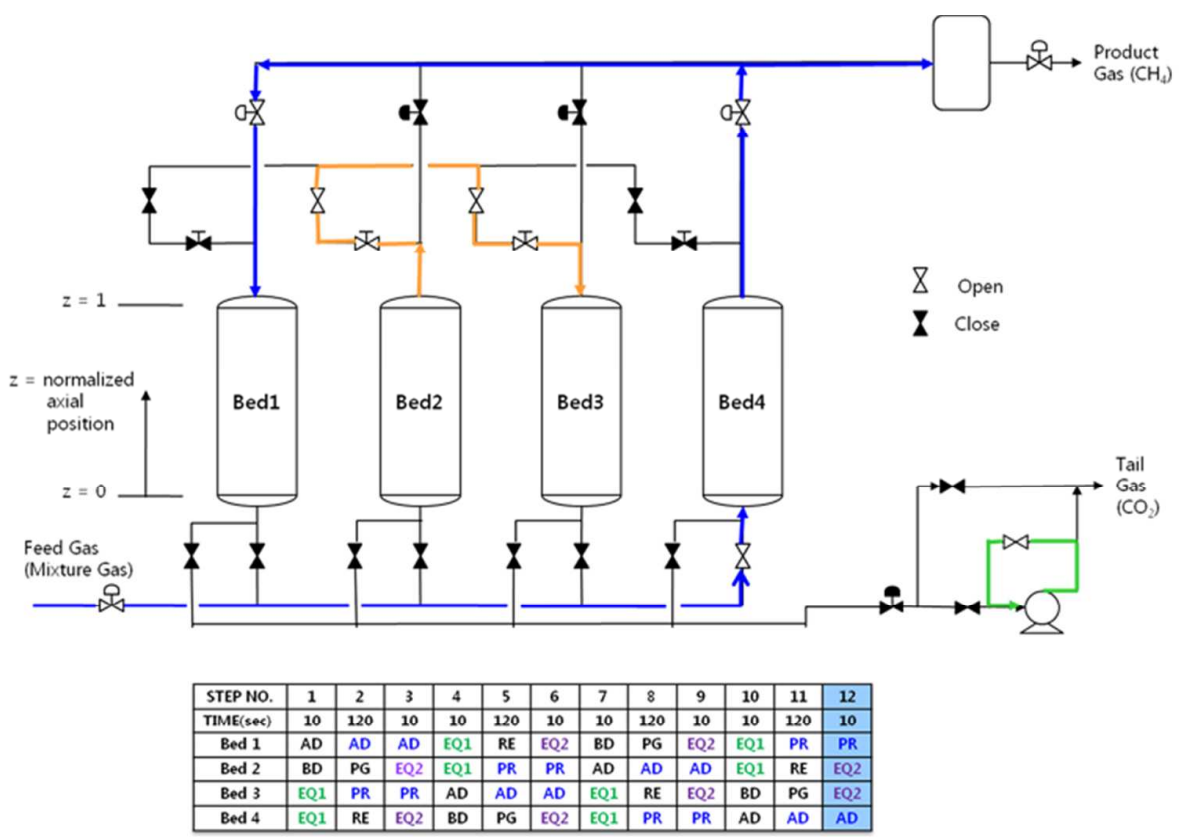

Figure S1.12. Operating Step 12 of Four-Bed VPSA 


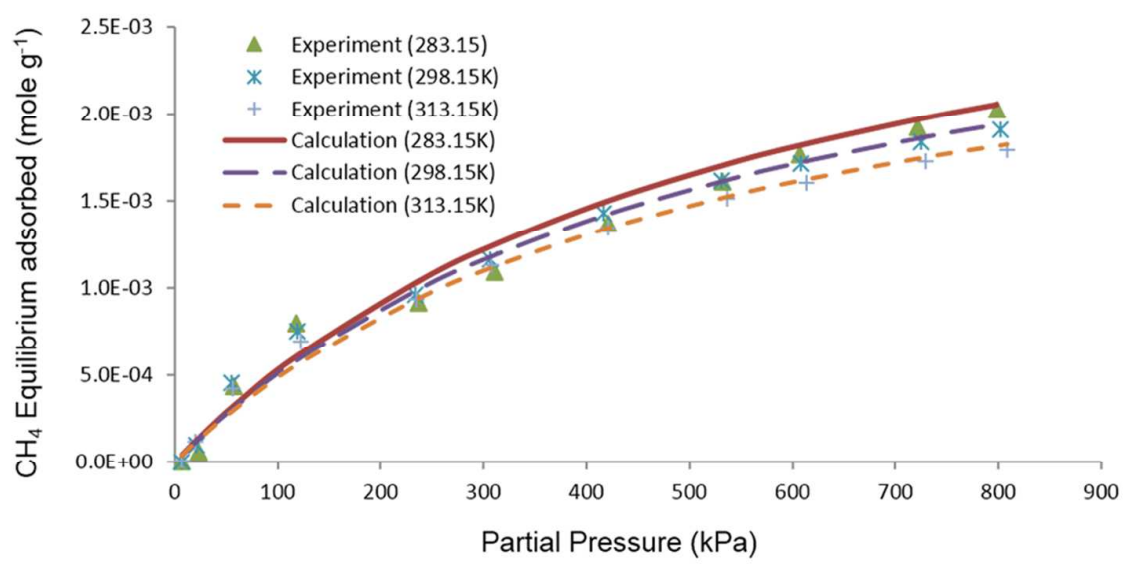

(a) $\mathrm{CH}_{4}$ adsorption isotherm

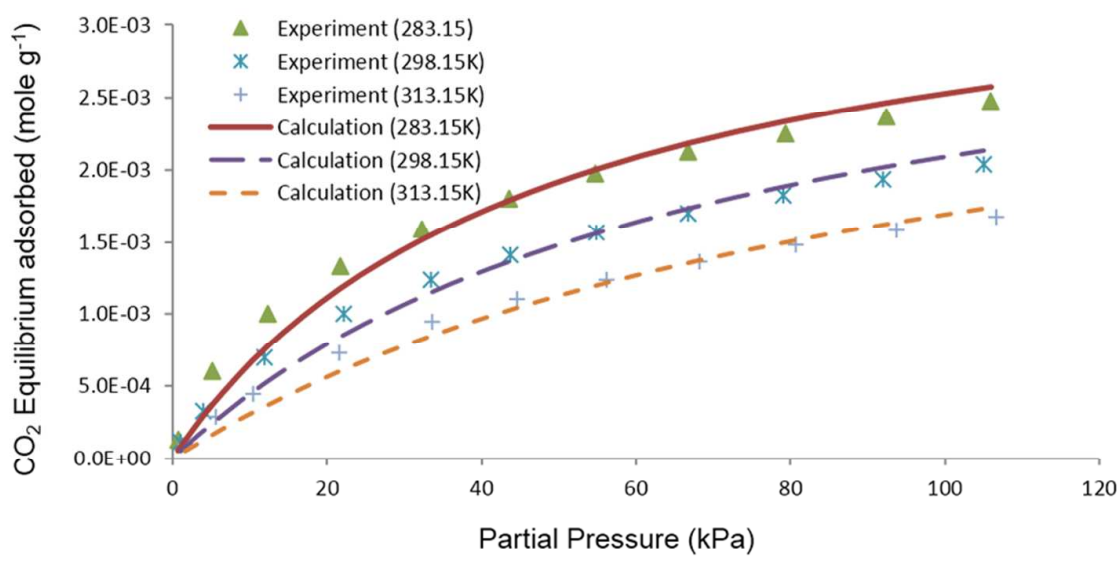

(b) $\mathrm{CO}_{2}$ adsorption isotherm

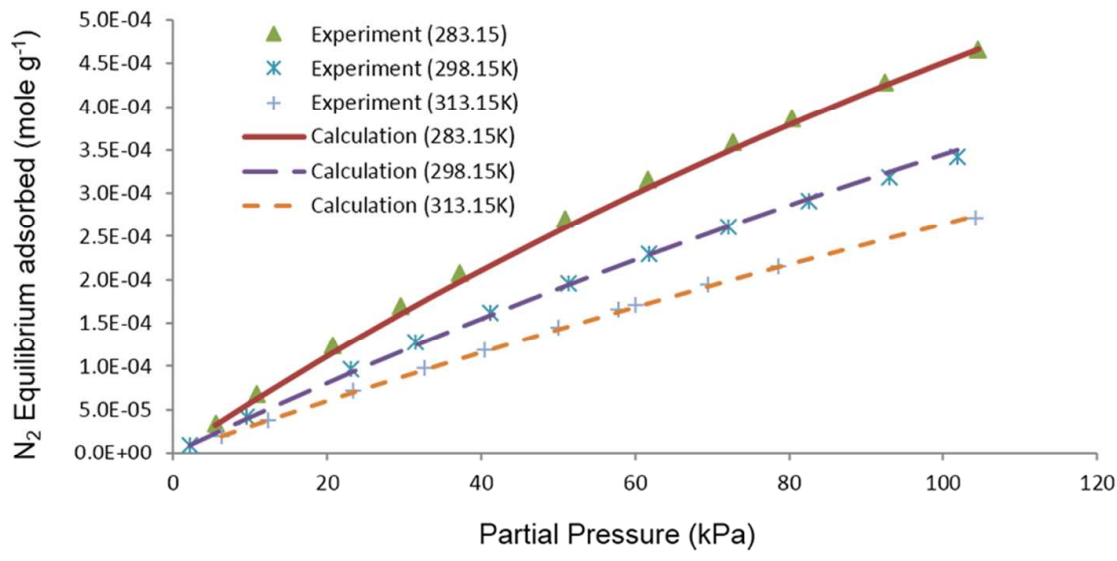

(c) $\mathrm{N}_{2}$ adsorption isotherm

Figure S2. Adsorption Isotherms of methane, carbon dioxide, and nitrogen (Ko, 2016) 


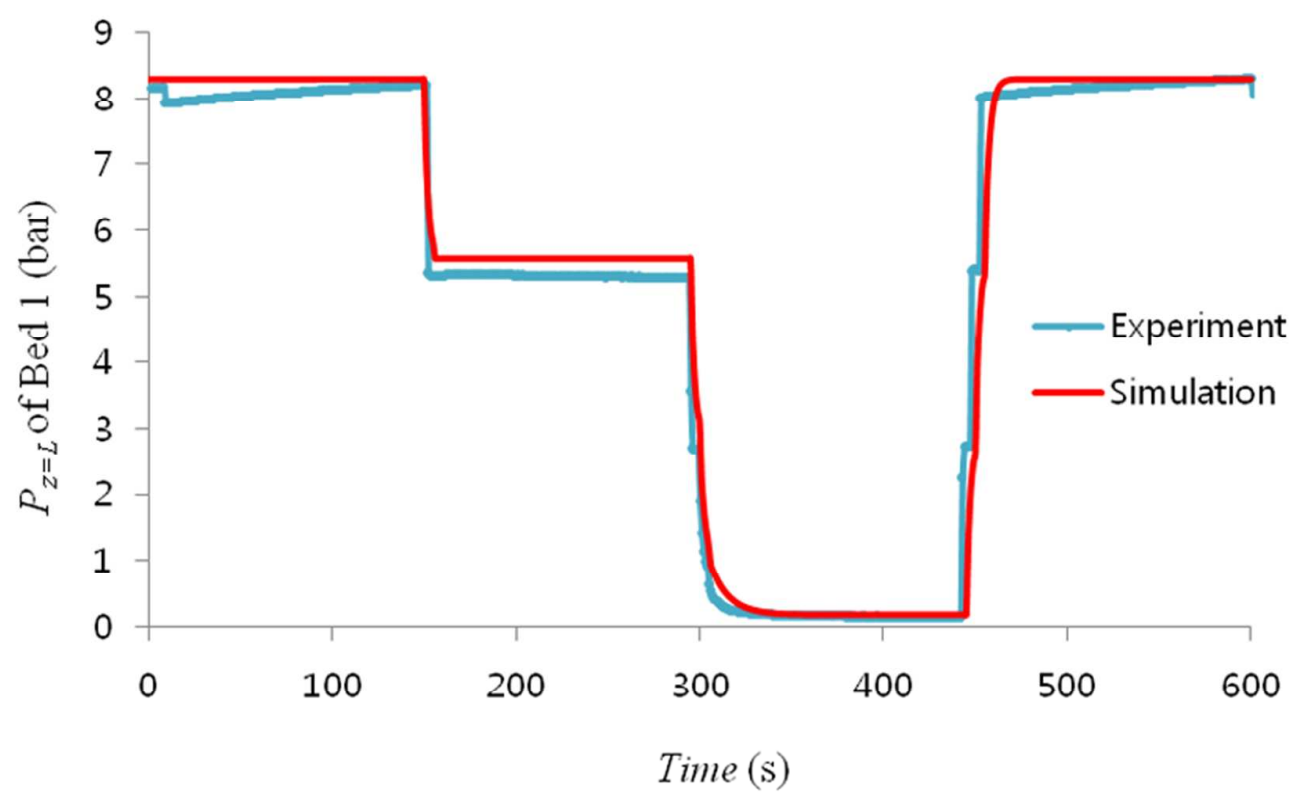

Figure S3. Pressure Profiles of Pilot Plant Operation Data and Simulation Result (Operating Steps and Times are shown in Table S2) 


\section{List of Supporting Tables}

Table S1. Comparison of Redlich-Kwong (RK) Equation of State with Ideal Gas Law in the Simulation (laboratory-scale \& pilot-scale)

Table S2. Operating Step of Pilot Plant Operation

Table S3. Comparison of nonadiabatic and adiabatic condition (laboratory-scale \& pilotscale)

Table S4. Optimal values of $P_{E Q}$ and $C_{V}$ in Cases $1 \mathrm{a}-1 \mathrm{c}$ (different constraints of methane purity and recovery at $293.15 \mathrm{~K}\left(T_{\text {feed }}=T_{\text {atm }}=T_{\text {wall }}\right)$

Table S5. Optimal values of $P_{E Q}$ and $C_{V}$ in Cases $2 \mathrm{a}-2 \mathrm{c}$ (different temperatures $\left.\left(T_{\text {feed }}=T_{\text {atm }}=T_{\text {wall }}\right)\right)$

Table S6. Optimal values of $P_{E Q}$ and $C_{V}$ in Cases $3 \mathrm{a}-3 \mathrm{c}$ (different adsorption times at $\left.293.15 \mathrm{~K}\left(T_{\text {feed }}=T_{\text {atm }}=T_{\text {wall }}\right)\right)$

Table S7. Optimal values of $P_{E Q}$ and $C_{V}$ in Cases $4 \mathrm{a}-4 \mathrm{c}$ at $293.15 \mathrm{~K}\left(T_{\text {feed }}=T_{\text {atm }}=T_{\text {wall }}\right)$

Table S8. Optimization Results of Cases 5a-5c

$\left(t_{A D}, P_{A D}\right.$, and $P_{P G}$ are added to decision variable set at $293.15 \mathrm{~K}\left(T_{\text {feed }}=T_{\text {atm }}=T_{\text {wall }}\right)$ and Obj $=10^{5} \varepsilon+$ Power $\left._{\text {sp,ave,Norm }}\right)$

Table S9. Optimization Results of Cases $6 \mathrm{a}-6 \mathrm{c}$

( $t_{A D}, P_{A D}$, and $P_{P G}$ are added to decision variable set at $293.15 \mathrm{~K}\left(T_{\text {feed }}=T_{\text {atm }}=T_{\text {wall }}\right)$ and Obj $=10^{5} \varepsilon-$ Purity $_{\mathrm{CH} 4}-$ Recovery $\left._{\mathrm{CH} 4}\right)$

Table S10. Optimization Results of Cases $7 \mathrm{a}-7 \mathrm{c}$

$\left(t_{A D}, P_{A D}\right.$, and $P_{P G}$ are added to decision variable set at $293.15 \mathrm{~K}\left(T_{\text {feed }}=T_{\text {atm }}=T_{\text {wall }}\right)$ and Obj $=10^{5} \varepsilon-$ Purity $_{\mathrm{CH} 4}-$ Recovery $_{\mathrm{CH} 4}+$ Power $\left._{\text {sp,ave, Norm }}\right)$ 
Table S1. Comparison of Redlich-Kwong (RK) Equation of State with Ideal Gas Law in the Simulation (laboratory-scale \& pilot-scale)

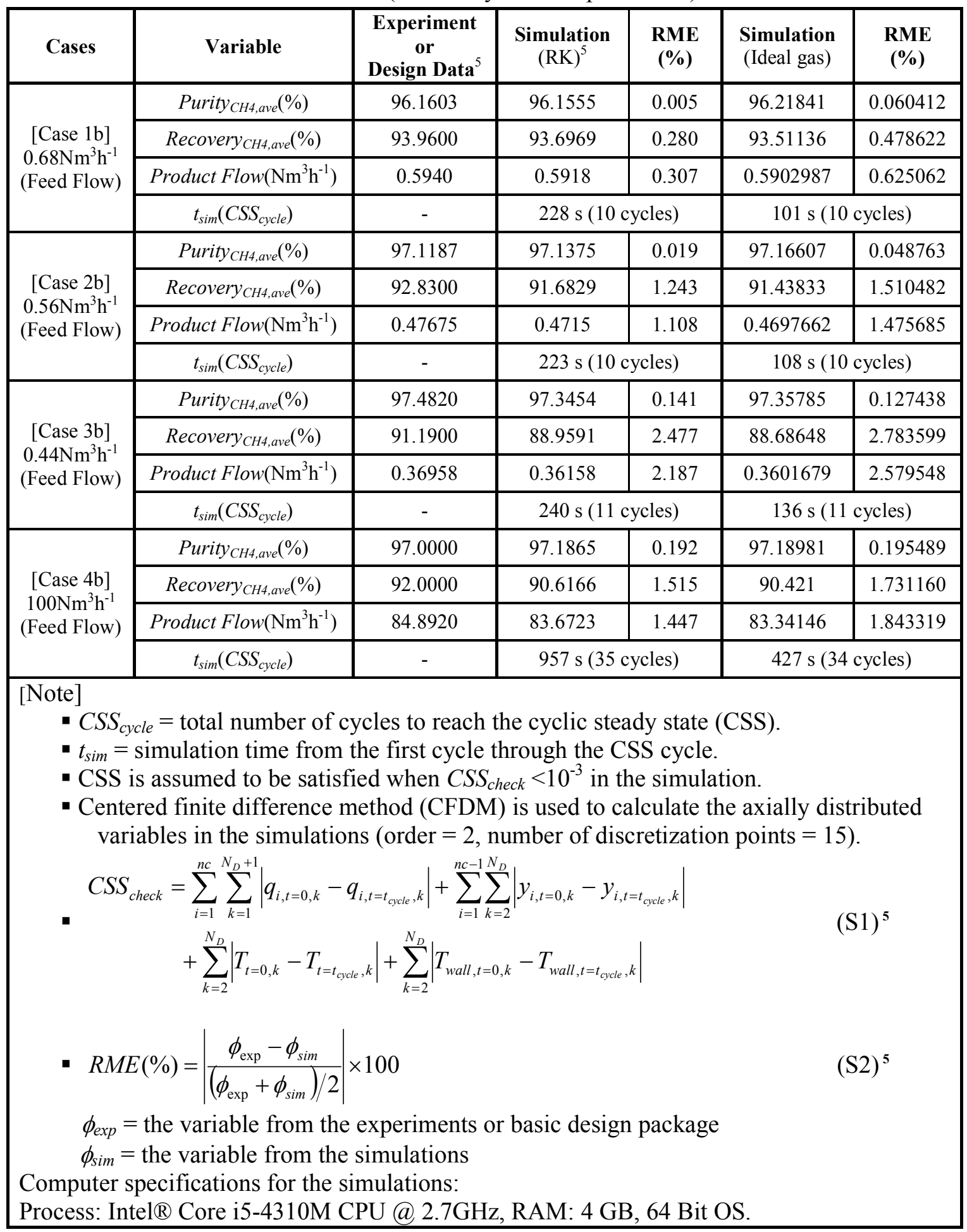


Table S2. Operating Step of Pilot Plant Operation

\begin{tabular}{|l|l|l|l|l|l|l|l|l|l|l|l|l|}
\hline Step No. & 1 & 2 & 3 & 4 & 5 & 6 & 7 & 8 & 9 & 10 & 11 & 12 \\
\hline Time (s) & 5 & 140 & 5 & 5 & 140 & 5 & 5 & 140 & 5 & 5 & 140 & 5 \\
\hline Bed 1 & AD & AD & AD & EQ1 & RE & EQ2 & BD & PG & EQ2 & EQ1 & PR & PR \\
\hline Bed 2 & BD & PG & EQ2 & EQ1 & PR & PR & AD & AD & AD & EQ1 & RE & EQ2 \\
\hline Bed 3 & EQ1 & PR & PR & AD & AD & AD & EQ1 & RE & EQ2 & BD & PG & EQ2 \\
\hline Bed 4 & EQ1 & RE & EQ2 & BD & PG & EQ2 & EQ1 & PR & PR & AD & AD & AD \\
\hline
\end{tabular}

AD: adsorption, EQ: pressure equalization, RE: repose, BD: blow down, PG: vacuum purge, PR: pressurization 
Table S3. Comparison of nonadiabatic and adiabatic condition (laboratory-scale \& pilot-scale)

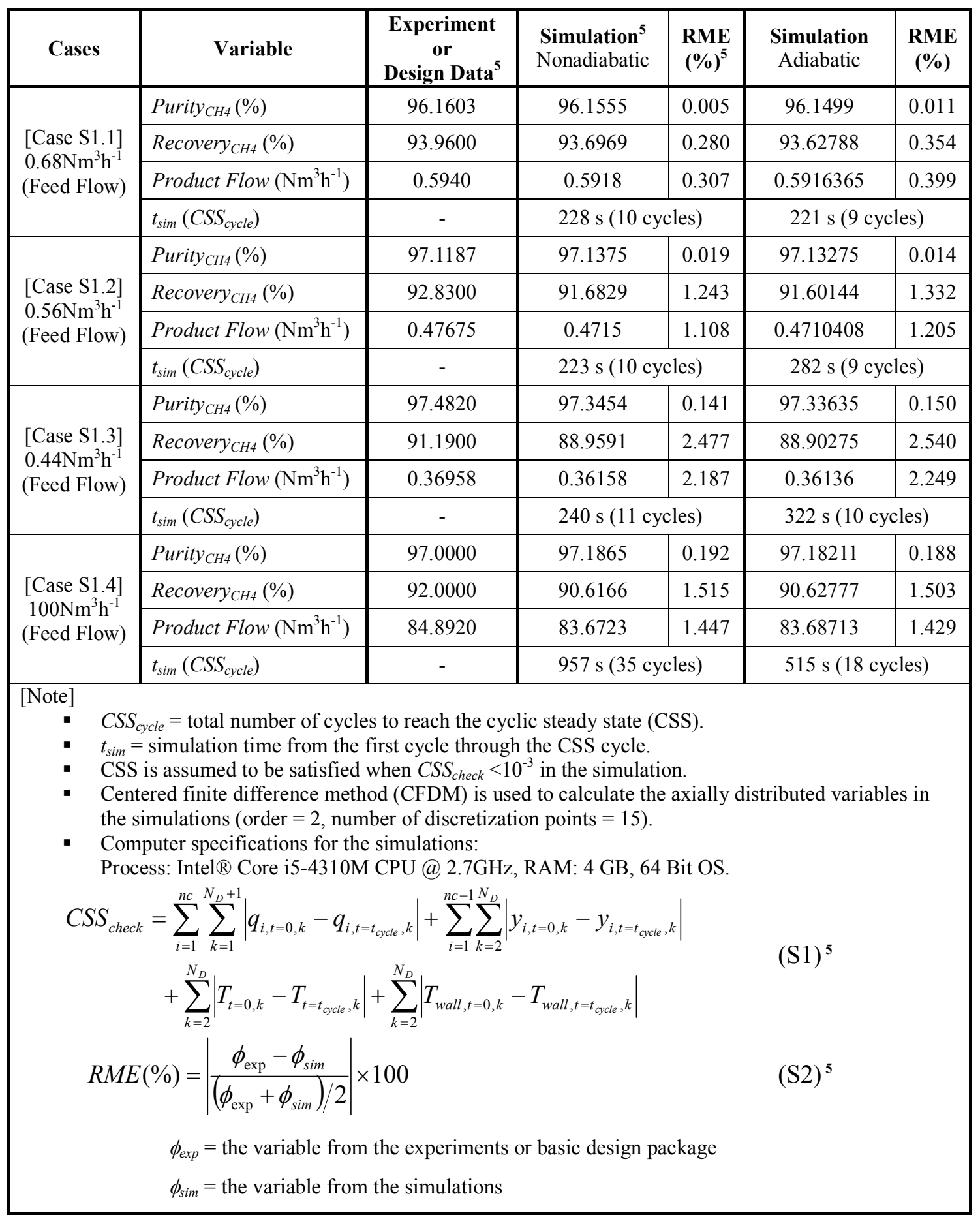


Table S4. Optimal values of $P_{E Q}$ and $C_{V}$ in Cases $1 \mathrm{a}-1 \mathrm{c}$ (different constraints of methane purity and recovery at $293.15 \mathrm{~K}\left(T_{\text {feed }}=T_{\text {atm }}=T_{\text {wall }}\right)$

\begin{tabular}{|c|c|c|c|}
\hline \multirow{2}{*}{ Variable } & $\begin{array}{c}\text { Case 1a } \\
\text { Purity }_{C_{4}} \geq 0.97 \\
\text { Recovey }_{C_{4}} \geq 0.9\end{array}$ & $\begin{array}{c}\text { Case 1b } \\
\text { Purity }_{C_{4}} \geq 0.975 \\
\text { Recovey }_{C_{4}} \geq 0.8\end{array}$ & $\begin{array}{c}\text { Case 1c } \\
\text { Purity }_{C_{4}} \geq 0.976 \\
\text { Recovey }_{C_{4}} \geq 0.75\end{array}$ \\
\hline$P_{E Q 1}^{\text {Step }(04)}(\mathrm{Pa})$ & $7.33346 \times 10^{5}$ & $7.33552 \times 10^{5}$ & $7.34031 \times 10^{5}$ \\
\hline$P_{E Q 2}^{\text {Step }(06)}(\mathrm{Pa})$ & $3.70743 \times 10^{5}$ & $3.70592 \times 10^{5}$ & $3.70812 \times 10^{5}$ \\
\hline$C_{V, E Q 1}^{\text {step }(04)}\left(\mathrm{m}^{3.5} / \mathrm{kg}^{0.5}\right)$ & $5.88503 \times 10^{-7}$ & $9.38319 \times 10^{-7}$ & $1.36688 \times 10^{-6}$ \\
\hline$C_{V, E Q 2}^{\text {step }(06)}\left(\mathrm{m}^{3.5} / \mathrm{kg}^{0.5}\right)$ & $1.0246 \times 10^{-6}$ & $1.63028 \times 10^{-6}$ & $2.37047 \times 10^{-6}$ \\
\hline$C_{V, B D}^{\text {step }(07)}\left(\mathrm{m}^{3.5} / \mathrm{kg}^{0.5}\right)$ & $1.85796 \times 10^{-6}$ & $2.95439 \times 10^{-6}$ & $4.29962 \times 10^{-6}$ \\
\hline$C_{V, P G}^{\text {step }(08)}\left(\mathrm{m}^{3.5} / \mathrm{kg}^{0.5}\right)$ & $1.17902 \times 10^{-6}$ & $1.87305 \times 10^{-6}$ & $2.72668 \times 10^{-6}$ \\
\hline$C_{V, E Q 2}^{\text {step }(09)}\left(\mathrm{m}^{3.5} / \mathrm{kg}^{0.5}\right)$ & $2.15478 \times 10^{-6}$ & $3.42938 \times 10^{-6}$ & $4.98422 \times 10^{-6}$ \\
\hline$C_{V, E Q 1}^{\text {step }(10)}\left(\mathrm{m}^{3.5} / \mathrm{kg}^{0.5}\right)$ & $8.53225 \times 10^{-7}$ & $1.35826 \times 10^{-6}$ & $1.97468 \times 10^{-6}$ \\
\hline$C_{V, P R}^{\text {steps }(11-12)}\left(\mathrm{m}^{3.5} / \mathrm{kg}^{0.5}\right)$ & $4.16267 \times 10^{-8}$ & $6.59288 \times 10^{-8}$ & $9.57137 \times 10^{-8}$ \\
\hline
\end{tabular}


Table S5. Optimal values of $P_{E Q}$ and $C_{V}$ in Cases $2 \mathrm{a}-2 \mathrm{c}$ (different temperatures $\left.\left(T_{\text {feed }}=T_{\text {atm }}=T_{\text {wall }}\right)\right)$

\begin{tabular}{|c|c|c|c|}
\hline Variable & $\begin{array}{c}\text { Case } 2 \mathbf{a} \\
\text { (=Case 1a) } \\
T=293.15 \mathrm{~K}\end{array}$ & $\begin{array}{c}\text { Case 2b } \\
T=303.15 \mathrm{~K}\end{array}$ & $\begin{array}{c}\text { Case 2c } \\
T=313.15 \mathrm{~K}\end{array}$ \\
\hline$P_{E Q 1}^{S t e p(04)} \quad(\mathrm{Pa})$ & $7.33346 \times 10^{5}$ & $7.33561 \times 10^{5}$ & $7.33800 \times 10^{5}$ \\
\hline$P_{E Q 2}^{S t e p(06)}(\mathrm{Pa})$ & $3.70743 \times 10^{5}$ & $3.70964 \times 10^{5}$ & $3.71185 \times 10^{5}$ \\
\hline$C_{V, E Q 1}^{\text {step(04) }}\left(\mathrm{m}^{3.5} / \mathrm{kg}^{0.5}\right)$ & $5.88503 \times 10^{-7}$ & $5.66816 \times 10^{-7}$ & $5.56062 \times 10^{-7}$ \\
\hline$C_{V, E Q 2}^{s t e p(06)}\left(\mathrm{m}^{3.5} / \mathrm{kg}^{0.5}\right)$ & $1.0246 \times 10^{-6}$ & $9.85598 \times 10^{-7}$ & $9.65515 \times 10^{-7}$ \\
\hline$C_{V, B D}^{s t e p(07)}\left(\mathrm{m}^{3.5} / \mathrm{kg}^{0.5}\right)$ & $1.85796 \times 10^{-6}$ & $1.78732 \times 10^{-6}$ & $1.75099 \times 10^{-6}$ \\
\hline$C_{V, P G}^{s t e p(08)}\left(\mathrm{m}^{3.5} / \mathrm{kg}^{0.5}\right)$ & $1.17902 \times 10^{-6}$ & $1.1344 \times 10^{-6}$ & $1.1116 \times 10^{-6}$ \\
\hline$C_{V, E Q 2}^{s t e p(09)}\left(\mathrm{m}^{3.5} / \mathrm{kg}^{0.5}\right)$ & $2.15478 \times 10^{-6}$ & $2.07102 \times 10^{-6}$ & $2.02727 \times 10^{-6}$ \\
\hline$C_{V, E Q 1}^{s t e p(10)}\left(\mathrm{m}^{3.5} / \mathrm{kg}^{0.5}\right)$ & $8.53225 \times 10^{-7}$ & $8.20909 \times 10^{-7}$ & $8.04341 \times 10^{-7}$ \\
\hline$C_{V, P R}^{\text {steps(11-12) }}\left(\mathrm{m}^{3.5} / \mathrm{kg}^{0.5}\right)$ & $4.16267 \times 10^{-8}$ & $4.01315 \times 10^{-8}$ & $3.93906 \times 10^{-8}$ \\
\hline
\end{tabular}


Table S6. Optimal values of $P_{E Q}$ and $C_{V}$ in Cases $3 \mathrm{a}-3 \mathrm{c}$ (different adsorption times at $\left.293.15 \mathrm{~K}\left(T_{\text {feed }}=T_{\text {atm }}=T_{\text {wall }}\right)\right)$

\begin{tabular}{|c|c|c|c|}
\hline Variable & $\begin{array}{c}\text { Case 3a } \\
t_{A D}^{\text {Steps }(01-03)}=120 \mathrm{~s}\end{array}$ & $\begin{array}{c}\text { Case 3b } \\
(=\text { Case 1a) } \\
t_{A D}^{\text {Steps }(01-03)}=140 s\end{array}$ & $\begin{array}{c}\text { Case 3c } \\
t_{A D}^{\text {Steps }(01-03)}=160 \mathrm{~s}\end{array}$ \\
\hline$P_{E Q 1}^{S t e p(04)}(\mathrm{Pa})$ & $7.33105 \times 10^{5}$ & $7.33346 \times 10^{5}$ & $7.33614 \times 10^{5}$ \\
\hline$P_{E Q 2}^{S t e p(06)}(\mathrm{Pa})$ & $3.70386 \times 10^{5}$ & $3.70743 \times 10^{5}$ & $3.71071 \times 10^{5}$ \\
\hline$C_{V, E Q 1}^{s t e p(04)}\left(\mathrm{m}^{3.5} / \mathrm{kg}^{0.5}\right)$ & $6.0092 \times 10^{-7}$ & $5.88503 \times 10^{-7}$ & $5.85415 \times 10^{-7}$ \\
\hline$C_{V, E Q 2}^{s t e p(06)}\left(\mathrm{m}^{3.5} / \mathrm{kg}^{0.5}\right)$ & $1.04594 \times 10^{-6}$ & $1.0246 \times 10^{-6}$ & $1.01922 \times 10^{-6}$ \\
\hline$C_{V, B D}^{s t e p(07)} \quad\left(\mathrm{m}^{3.5} / \mathrm{kg}^{0.5}\right)$ & $1.89525 \times 10^{-6}$ & $1.85796 \times 10^{-6}$ & $1.84949 \times 10^{-6}$ \\
\hline$C_{V, P G}^{s t e p(08)} \quad\left(\mathrm{m}^{3.5} / \mathrm{kg}^{0.5}\right)$ & $1.2037 \times 10^{-6}$ & $1.17902 \times 10^{-6}$ & $1.17339 \times 10^{-6}$ \\
\hline$C_{V, E Q 2}^{s t e p(09)}\left(\mathrm{m}^{3.5} / \mathrm{kg}^{0.5}\right)$ & $2.20093 \times 10^{-6}$ & $2.15478 \times 10^{-6}$ & $2.14251 \times 10^{-6}$ \\
\hline$C_{V, E Q 1}^{s t e p(10)}\left(\mathrm{m}^{3.5} / \mathrm{kg}^{0.5}\right)$ & $8.71286 \times 10^{-7}$ & $8.53225 \times 10^{-7}$ & $8.48559 \times 10^{-7}$ \\
\hline$C_{V, P R}^{s t e p s(11-12)}\left(\mathrm{m}^{3.5} / \mathrm{kg}^{0.5}\right)$ & $4.43948 \times 10^{-8}$ & $4.16267 \times 10^{-8}$ & $4.02787 \times 10^{-8}$ \\
\hline
\end{tabular}


Table S7. Optimal values of $P_{E Q}$ and $C_{V}$ in Cases $4 \mathrm{a}-4 \mathrm{c}$ at $293.15 \mathrm{~K}\left(T_{\text {feed }}=T_{\text {atm }}=T_{\text {wall }}\right)$

\begin{tabular}{|c|c|c|c|}
\hline Variable & $\begin{array}{c}\text { Case 4a } \\
\text { Purity }_{\mathrm{CH}_{4}} \geq 0.975 \\
\text { Recovey }_{\mathrm{CH}_{4}} \geq 0.9\end{array}$ & $\begin{array}{c}\text { Case 4b } \\
\text { Purity }_{\mathrm{CH}_{4}} \geq 0.9755 \\
\text { Recovey }_{\mathrm{CH}_{4}} \geq 0.88\end{array}$ & $\begin{array}{c}\text { Case 4c } \\
\text { Purity }_{\mathrm{CH}_{4}} \geq 0.976 \\
\text { Recovey }_{\mathrm{CH}_{4}} \geq 0.85\end{array}$ \\
\hline$P_{E Q 1}^{S t e p(04)}(\mathrm{Pa})$ & $7.48146 \times 10^{5}$ & $6.85474 \times 10^{5}$ & $6.00061 \times 10^{5}$ \\
\hline$P_{E Q 2}^{S t e p(06)}(\mathrm{Pa})$ & $3.78221 \times 10^{5}$ & $3.46997 \times 10^{5}$ & $3.02110 \times 10^{5}$ \\
\hline$C_{V, E Q 1}^{\text {step }(04)}\left(\mathrm{m}^{3.5} / \mathrm{kg}^{0.5}\right)$ & $7.47332 \times 10^{-7}$ & $1.07602 \times 10^{-6}$ & $1.63412 \times 10^{-6}$ \\
\hline$C_{V, E Q^{2}}^{\text {step }(06)}\left(\mathrm{m}^{3.5} / \mathrm{kg}^{0.5}\right)$ & $1.3006 \times 10^{-6}$ & $1.86803 \times 10^{-6}$ & $2.8406 \times 10^{-6}$ \\
\hline$C_{V, B D}^{\text {step }(07)}\left(\mathrm{m}^{3.5} / \mathrm{kg}^{0.5}\right)$ & $2.37674 \times 10^{-6}$ & $3.27057 \times 10^{-6}$ & $4.59118 \times 10^{-6}$ \\
\hline$C_{V, P G}^{s t e p(08)}\left(\mathrm{m}^{3.5} / \mathrm{kg}^{0.5}\right)$ & $1.51055 \times 10^{-6}$ & $2.07661 \times 10^{-6}$ & $3.19929 \times 10^{-6}$ \\
\hline$C_{V, E Q 2}^{s t e p(09)}\left(\mathrm{m}^{3.5} / \mathrm{kg}^{0.5}\right)$ & $2.73576 \times 10^{-6}$ & $3.91933 \times 10^{-6}$ & $6.00809 \times 10^{-6}$ \\
\hline$C_{V, E Q 1}^{s t e p(10)} \quad\left(\mathrm{m}^{3.5} / \mathrm{kg}^{0.5}\right)$ & $1.08287 \times 10^{-6}$ & $1.55609 \times 10^{-6}$ & $2.36341 \times 10^{-6}$ \\
\hline$C_{V, P R}^{\text {steps }(11-12)}\left(\mathrm{m}^{3.5} / \mathrm{kg}^{0.5}\right)$ & $5.14875 \times 10^{-8}$ & $7.23213 \times 10^{-8}$ & $1.08035 \times 10^{-7}$ \\
\hline
\end{tabular}


Table S8. Optimization Results of Cases $5 \mathrm{a}-5 \mathrm{c}$

( $t_{A D}, P_{A D}$, and $P_{P G}$ are added to decision variable set at $293.15 \mathrm{~K}\left(T_{\text {feed }}=T_{\text {atm }}=T_{\text {wall }}\right)$ and Obj $=10^{5} \varepsilon+$ Power $_{s p, a v e, N o r m}$ )

\begin{tabular}{|c|c|c|c|}
\hline Variable & $\begin{array}{c}\text { Case 5a } \\
\text { Purity }_{\mathrm{CH} 4} \geq 97.5 \% \\
\text { Recovery }_{\mathrm{CH} 4} \geq 90 \% \\
\end{array}$ & $\begin{array}{l}\text { Case 5b } \\
\text { Purity }_{\mathrm{CH} 4} \geq 97.55 \% \\
\text { Recovery }_{\mathrm{CH} 4} \geq 88 \% \\
\end{array}$ & $\begin{array}{c}\text { Case 5c } \\
\text { Purity }_{\mathrm{CH} 4} \geq 97.6 \% \\
\text { Recovery }_{\mathrm{CH} 4} \geq 85 \% \\
\end{array}$ \\
\hline$t_{A D}{ }^{\text {Step }(02)}(\mathrm{s})$ & 180 & 180 & 180 \\
\hline$t_{A D}{ }_{S t e p(01-03)}(\mathrm{s})$ & 200 & 200 & 200 \\
\hline Purity $_{\mathrm{CH} 4}(\%)$ & 97.5 & 97.55 & 97.6 \\
\hline Recovery $_{\mathrm{CH} 4}(\%)$ & 94.7 & 90.8221 & 85.0 \\
\hline Purity $_{\mathrm{CO} 2}(\%)$ & 0.0240 & 0.0134 & $3.1804 \times 10^{-5}$ \\
\hline Recovery $\mathrm{CO}_{2}(\%)$ & 0.2551 & 0.0137 & $3.0351305 \times 10^{-4}$ \\
\hline$L_{\text {bed }}(\mathrm{m})$ & 1.5 & 1.0 & 1.0 \\
\hline$t_{\text {cont }}(\mathrm{s})$ & 1.23058 & 1.87855 & 2.84524 \\
\hline$P_{A D}^{\text {Step }(01-03)}(\mathrm{Pa})$ & $9.0 \times 10^{5}$ & $9.0 \times 10^{5}$ & $9.0 \times 10^{5}$ \\
\hline$P_{B D}{ }^{\text {Step }(07)}(\mathrm{Pa})$ & $1.0 \times 10^{5}$ & $1.0 \times 10^{5}$ & $1.0 \times 10^{5}$ \\
\hline$P_{P G}{ }^{\operatorname{Step}(08)}(\mathrm{Pa})$ & $5.0 \times 10^{3}$ & $5.0 \times 10^{3}$ & 5442.8125 \\
\hline$D_{\text {inside }}(\mathrm{m})$ & $8.9159 \times 10^{-2}$ & 0.1349 & 0.1660 \\
\hline $\begin{array}{c}\text { Specific Power }_{\text {ave }} \\
(\mathrm{J} / \mathrm{mol})\end{array}$ & 8033.4736 & 8403.965 & 9014.254 \\
\hline$P_{E Q 1}^{\text {Step }(04)}(\mathrm{Pa})$ & $5.99884 \times 10^{5}$ & $5.96616 \times 10^{5}$ & $6.00062 \times 10^{5}$ \\
\hline$P_{E Q 2}^{S t e p(06)}(\mathrm{Pa})$ & $3.02208 \times 10^{5}$ & $3.1807 \times 10^{5}$ & $3.02110 \times 10^{5}$ \\
\hline$C_{V, E Q 1}^{s t e p(04)}\left(\mathrm{m}^{3.5} / \mathrm{kg}^{0.5}\right)$ & $7.04279 \times 10^{-7}$ & $1.07691 \times 10^{-6}$ & $1.63412 \times 10^{-6}$ \\
\hline$C_{V, E Q 2}^{s t e p(06)}\left(\mathrm{m}^{3.5} / \mathrm{kg}^{0.5}\right)$ & $1.22833 \times 10^{-6}$ & $1.87692 \times 10^{-6}$ & $2.8406 \times 10^{-6}$ \\
\hline$C_{V, B D}^{\text {step(07) }}\left(\mathrm{m}^{3.5} / \mathrm{kg}^{0.5}\right)$ & $1.98742 \times 10^{-6}$ & $3.03159 \times 10^{-6}$ & $4.59121 \times 10^{-6}$ \\
\hline$C_{V, P G}^{s t e p(08)}\left(\mathrm{m}^{3.5} / \mathrm{kg}^{0.5}\right)$ & $1.39719 \times 10^{-6}$ & $2.12943 \times 10^{-6}$ & $3.19929 \times 10^{-6}$ \\
\hline$C_{V, E Q 2}^{s t e p(09)}\left(\mathrm{m}^{3.5} / \mathrm{kg}^{0.5}\right)$ & $2.60033 \times 10^{-6}$ & $3.97459 \times 10^{-6}$ & $6.00808 \times 10^{-6}$ \\
\hline$C_{V, E Q 1}^{s t e p(10)}\left(\mathrm{m}^{3.5} / \mathrm{kg}^{0.5}\right)$ & $1.02164 \times 10^{-6}$ & $1.56126 \times 10^{-6}$ & $2.36341 \times 10^{-6}$ \\
\hline$C_{V, P R}^{\text {steps }(11-12)}\left(\mathrm{m}^{3.5} / \mathrm{kg}^{0.5}\right)$ & $4.71658 \times 10^{-8}$ & $7.16445 \times 10^{-8}$ & $1.08036 \times 10^{-7}$ \\
\hline Obj & 0.0803 & 0.0840 & 0.0901 \\
\hline Total CPU time (s) & 968.439 & 503.4 & 846.165 \\
\hline \multicolumn{4}{|c|}{$\begin{array}{l}\text { Constraints of Cases } 5 \mathrm{a}-5 \mathrm{c} \text { are same as that of Case } 4 \mathrm{c} \\
\text { Computer specifications for the optimizations: } \\
\text { Process: Intel® Core i5-4310M CPU @ } 2.7 \mathrm{GHz}, \mathrm{RAM}: 4 \mathrm{~GB}, 64 \text { Bit OS. }\end{array}$} \\
\hline
\end{tabular}


Table S9. Optimization Results of Cases $6 \mathrm{a}-6 \mathrm{c}$

$\left(t_{A D}, P_{A D}\right.$, and $P_{P G}$ are added to decision variable set at $293.15 \mathrm{~K}\left(T_{\text {feed }}=T_{\text {atm }}=T_{\text {wall }}\right)$ and Obj $=10^{5} \varepsilon-$ Purity $_{\mathrm{CH} 4}-$ Recovery $\left._{\mathrm{CH} 4}\right)$

\begin{tabular}{|c|c|c|c|}
\hline Variable & $\begin{array}{c}\text { Case 6a } \\
\text { Purity }_{\mathrm{CH} 4} \geq 97.5 \% \\
\text { Recovery }_{\mathrm{CH} 4} \geq 90 \% \\
\end{array}$ & $\begin{array}{c}\text { Case 6b } \\
\text { Purity }_{\mathrm{CH} 4} \geq 97.55 \% \\
\text { Recovery }_{\mathrm{CH} 4} \geq 88 \% \\
\end{array}$ & $\begin{array}{c}\text { Case 6c } \\
\text { Purity }_{\mathrm{CH} 4} \geq 97.6 \% \\
\text { Recovery }_{\mathrm{CH} 4} \geq 85 \% \\
\end{array}$ \\
\hline$t_{A D}^{\text {Step }(02)}(\mathrm{s})$ & 180 & 180 & 180 \\
\hline$t_{A D}{ }^{\text {Step }(01-03)}(\mathrm{s})$ & 200 & 200 & 200 \\
\hline Purity $_{\mathrm{CH} 4}(\%)$ & 97.5 & 97.55 & 97.6 \\
\hline Recovery $_{\mathrm{CH}}(\%)$ & 94.6912 & 90.8664 & 85.0227 \\
\hline Purity $_{\mathrm{CO} 2}(\%)$ & 0.0227 & $1.2798 \mathrm{E}-3$ & $1.5325 \times 10^{-5}$ \\
\hline Recovery $_{\mathrm{CO} 2}(\%)$ & 0.2417 & 0.1306 & $1.4628 \times 10^{-4}$ \\
\hline$L_{\text {bed }}(\mathrm{m})$ & 1.41114 & 1.5 & 1.0 \\
\hline$t_{\text {cont }}(\mathrm{s})$ & 1.20615 & 1.8639 & 2.8374 \\
\hline$P_{A D}^{\text {Step }(01-03)}(\mathrm{Pa})$ & $9.5535 \times 10^{5}$ & $9.0 \times 10^{5}$ & $9.0 \times 10^{5}$ \\
\hline$P_{B D}{ }^{S t e p(07)}(\mathrm{Pa})$ & $1.0 \times 10^{5}$ & $1.0 \times 10^{5}$ & $1.0 \times 10^{5}$ \\
\hline$P_{P G}^{S t e p(08)}(\mathrm{Pa})$ & $5.0 \times 10^{3}$ & $5.0 \times 10^{3}$ & $5.0 \times 10^{3}$ \\
\hline$D_{\text {inside }}(\mathrm{m})$ & 0.0910 & 0.1097 & 0.1658 \\
\hline $\begin{array}{c}\text { Specific Power ave } \\
(\mathrm{J} / \mathrm{mol})\end{array}$ & 8306.942 & 8399.462 & 9017.869 \\
\hline$P_{E Q 1}^{\text {Step }(04)}(\mathrm{Pa})$ & $6.36610 \times 10^{5}$ & $6.00085 \times 10^{5}$ & $5.99924 \times 10^{5}$ \\
\hline$P_{E Q 2}^{S t e p(06)}(\mathrm{Pa})$ & $3.20546 \times 10^{5}$ & $3.02207 \times 10^{5}$ & $3.01825 \times 10^{5}$ \\
\hline$C_{V, E Q 1}^{s t e p(04)}\left(\mathrm{m}^{3.5} / \mathrm{kg}^{0.5}\right)$ & $6.69578 \times 10^{-7}$ & $1.06803 \times 10^{-6}$ & $1.6302 \times 10^{-6}$ \\
\hline$C_{V, E Q 2}^{s t e p(06)}\left(\mathrm{m}^{3.5} / \mathrm{kg}^{0.5}\right)$ & $1.16876 \times 10^{-6}$ & $1.86054 \times 10^{-6}$ & $2.83507 \times 10^{-6}$ \\
\hline$C_{V, B D}^{s t e p(07)}\left(\mathrm{m}^{3.5} / \mathrm{kg}^{0.5}\right)$ & $1.95625 \times 10^{-6}$ & $3.00838 \times 10^{-6}$ & $4.57811 \times 10^{-6}$ \\
\hline$C_{V, P G}^{s t e p(08)}\left(\mathrm{m}^{3.5} / \mathrm{kg}^{0.5}\right)$ & $1.36911 \times 10^{-6}$ & $2.11324 \times 10^{-6}$ & $3.21469 \times 10^{-6}$ \\
\hline$C_{V, E Q 2}^{s t e p(09)} \quad\left(\mathrm{m}^{3.5} / \mathrm{kg}^{0.5}\right)$ & $2.47664 \times 10^{-6}$ & $3.93897 \times 10^{-6}$ & $6.00331 \times 10^{-6}$ \\
\hline$C_{V, E Q 1}^{s t e p(10)} \quad\left(\mathrm{m}^{3.5} / \mathrm{kg}^{0.5}\right)$ & $9.71813 \times 10^{-7}$ & $1.54749 \times 10^{-6}$ & $2.35834 \times 10^{-6}$ \\
\hline$C_{V, P R}^{\text {steps(11-12) }}\left(\mathrm{m}^{3.5} / \mathrm{kg}^{0.5}\right)$ & $4.48342 \times 10^{-8}$ & $7.12571 \times 10^{-8}$ & $1.07777 \times 10^{-7}$ \\
\hline Obj & -1.92191 & -1.8842 & -1.8262 \\
\hline Total CPU time (s) & 3063.83 & 1472.49 & 284.078 \\
\hline \multicolumn{4}{|c|}{ 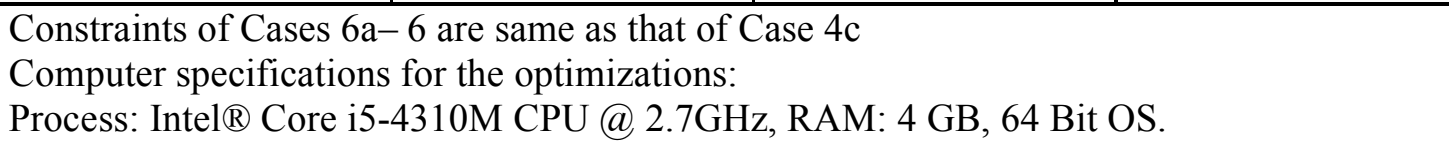 } \\
\hline
\end{tabular}


Table S10. Optimization Results of Cases $7 \mathrm{a}-7 \mathrm{c}$

( $t_{A D}, P_{A D}$, and $P_{P G}$ are added to decision variable set at $293.15 \mathrm{~K}\left(T_{\text {feed }}=T_{\text {atm }}=T_{\text {wall }}\right)$ and Obj $=10^{5} \varepsilon-$ Purity $_{\mathrm{CH} 4}-$ Recovery $_{\mathrm{CH} 4}+$ Power $\left._{\text {sp, ave,Norm }}\right)$

\begin{tabular}{|c|c|c|c|}
\hline Variable & $\begin{array}{c}\text { Case 7a } \\
\text { Purity }_{\mathrm{CH} 4} \geq 97.5 \% \\
\text { Recovery }_{\mathrm{CH} 4} \geq 90 \% \\
\end{array}$ & $\begin{array}{c}\text { Case 7b } \\
\text { Purity }_{\mathrm{CH} 4} \geq 97.55 \% \\
\text { Recovery }_{\mathrm{CH} 4} \geq 88 \% \\
\end{array}$ & $\begin{array}{c}\text { Case 7c } \\
\text { Purity }_{\mathrm{CH} 4} \geq 97.6 \% \\
\text { Recovery }_{\mathrm{CH} 4} \geq 85 \% \\
\end{array}$ \\
\hline$t_{A D}{ }^{\text {Step }(02)}(\mathrm{s})$ & 180 & 180 & 180 \\
\hline$t_{A D}{ }^{\text {Step }(01-03)}(\mathrm{s})$ & 200 & 200 & 200 \\
\hline Purity $_{\mathrm{CH} 4}(\%)$ & 97.5 & 97.55 & 97.6 \\
\hline Recovery ${ }_{C H 4}(\%)$ & 94.7 & 90.8666 & 85.0227 \\
\hline Purity $_{\mathrm{CO} 2}(\%)$ & 0.0240 & $1.2799 \times 10^{-3}$ & $1.5325 \times 10^{-5}$ \\
\hline Recovery ${ }_{\mathrm{CO} 2}(\%)$ & 0.2551 & 0.0131 & $1.4629 \times 10^{-4}$ \\
\hline$L_{\text {bed }}(\mathrm{m})$ & 1.5 & 1.5 & 1.0 \\
\hline$t_{\text {cont }}(\mathrm{s})$ & 1.23058 & 1.86382 & 2.83738 \\
\hline$P_{A D}^{\text {Step(01-03) }}(\mathrm{Pa})$ & $9.0 \times 10^{5}$ & $9.0 \times 10^{5}$ & $9.0 \times 10^{5}$ \\
\hline$P_{B D}^{\text {Step }(07)}(\mathrm{Pa})$ & $1.0 \times 10^{5}$ & $1.0 \times 10^{5}$ & $1.0 \times 10^{5}$ \\
\hline$P_{P G}^{\text {Step }(08)}(\mathrm{Pa})$ & $5 \times 10^{3}$ & $5 \times 10^{3}$ & $5 \times 10^{3}$ \\
\hline$D_{\text {inside }}(\mathrm{m})$ & $8.9160 \times 10^{-2}$ & 0.109727 & 0.165812 \\
\hline $\begin{array}{c}\text { Specific Power ave } \\
(\mathrm{J} / \mathrm{mol})\end{array}$ & 8033.47 & 8399.44 & 9017.87 \\
\hline$P_{E Q 1}^{\text {Step }(04)}(\mathrm{Pa})$ & $5.99884 \times 10^{5}$ & $6.00085 \times 10^{5}$ & $5.99924 \times 10^{5}$ \\
\hline$P_{E Q 2}^{\text {Step }(06)}(\mathrm{Pa})$ & $3.02208 \times 10^{5}$ & $3.02207 \times 10^{5}$ & $3.01825 \times 10^{5}$ \\
\hline$C_{V, E Q 1}^{\text {step }(04)}\left(\mathrm{m}^{3.5} / \mathrm{kg}^{0.5}\right)$ & $7.0428 \times 10^{-7}$ & $1.06802 \times 10^{-6}$ & $1.6302 \times 10^{-6}$ \\
\hline$C_{V, E Q 2}^{s t e p(06)}\left(\mathrm{m}^{3.5} / \mathrm{kg}^{0.5}\right)$ & $1.22834 \times 10^{-6}$ & $1.86052 \times 10^{-6}$ & $2.83507 \times 10^{-6}$ \\
\hline$C_{V, B D}^{s t e p(07)}\left(\mathrm{m}^{3.5} / \mathrm{kg}^{0.5}\right)$ & $1.98742 \times 10^{-6}$ & $3.00833 \times 10^{-6}$ & $4.57809 \times 10^{-6}$ \\
\hline$C_{V, P G}^{s t e p(08)}\left(\mathrm{m}^{3.5} / \mathrm{kg}^{0.5}\right)$ & $1.3972 \times 10^{-6}$ & $2.11317 \times 10^{-6}$ & $3.21472 \times 10^{-6}$ \\
\hline$C_{V, E Q 2}^{\text {step }(09)}\left(\mathrm{m}^{3.5} / \mathrm{kg}^{0.5}\right)$ & $2.60035 \times 10^{-6}$ & $3.98389 \times 10^{-6}$ & $6.0033 \times 10^{-6}$ \\
\hline$C_{V, E Q 1}^{s t e p(10)}\left(\mathrm{m}^{3.5} / \mathrm{kg}^{0.5}\right)$ & $1.02164 \times 10^{-6}$ & $1.54746 \times 10^{-6}$ & $2.35834 \times 10^{-6}$ \\
\hline$C_{V, P R}^{\text {steps(11-12) }}\left(\mathrm{m}^{3.5} / \mathrm{kg}^{0.5}\right)$ & $4.71658 \times 10^{-8}$ & $7.12558 \times 10^{-8}$ & $1.07777 \times 10^{-7}$ \\
\hline Obj & -1.84166 & -1.80017 & -1.73605 \\
\hline Total CPU time (s) & 1811.19 & 1037.27 & 341.034 \\
\hline \multicolumn{4}{|c|}{$\begin{array}{l}\text { Constraints of Cases } 6 \mathrm{a}-6 \text { are same as that of Case } 4 \mathrm{c} \\
\text { Computer specifications for the optimizations: } \\
\text { Process: Intel® Core i5-4310M CPU @ 2.7GHz, RAM: } 4 \text { GB, } 64 \text { Bit OS. }\end{array}$} \\
\hline
\end{tabular}

